\title{
Influence of the form of axisymmetric load on the stress-strain state of an elastoplastic half-space
}

\author{
Peter Ogar ${ }^{1, *}$, Denis Gorokhov ${ }^{1}$, Leonid Mamaev $^{1}$, and Vladislav Kushnarev ${ }^{1}$ \\ ${ }^{1}$ Bratsk State University, Makarenko 40, 665709 Bratsk, Russia
}

\begin{abstract}
The problems of elastic sinking of materials and elastic restoration of an imprint under loading of an elastic-plastic half-space with an axisymmetric load of the form $p(r)=p_{0}\left(1-r^{2} / a^{2}\right)^{\beta}$, where $0<\beta<0.5$ are considered. Expressions are obtained that describing the stress-strain state of an elastoplastic half-space. The conditions of the onset of plastic deformation in the near-surface layer and on the half-space of surface are considered depending on the parameter $\beta$.
\end{abstract}

\section{Introduction}

Many models of rough surfaces are represented by asperities in the form of a set of spherical segments with the same radii [1, 2, 3, etc.]. In most cases, the contact of metal rough surfaces is elastoplastic [4]. However at present, there is no analytical solution to the contact problem for an elastoplastic force indentation [5, 6]. As indicated in recent reviews $[7,8]$, many analytical, experimental, and numerical studies have been performed in the past to model and predict the properties of an elastoplastic contact. However because of their complexity, a closed solution has not been proposed. The authors of $[5,6]$ attribute this to the difficulties of taking into account the hardenability of the material during loading. Other authors find the reason for the difficulties in describing the effects of "pileup / sink-in" $[9,10,11,12$, etc.]. There are also problems in describing the contact of bodies with similar physical and mechanical properties [5, 13, 14, 15, etc.]. To simplify the complex solution of these problems, most authors model the contact of the sphere and halfspace. Moreover, contact models can be divided into two main groups: interstitial models (rigid or elastic sphere) and flattening models (rigid half-space) [13].

When a sphere is introducted into a deformable half-space, an elastic region, a region of limited elastic elasticity and a region of developed elastic elasticity are distinguished [4, 7]. They differ in the ratio of the role of two independent processes: elastic pushing of materials (sink-in) and plastic displacement of material (pile-up). In our view of the above problems of studying elastoplasticity, we can include the issues of elastic sinking of materials and elastic restoration of the imprint due to their practical importance. Their solution is relevant when using the kinetic indentation diagram to describe the elastoplastic contact indentation [16], when determining the joint density of rough surfaces [17, 18], when determining contact characteristics when unloading a pre-loaded joint [19].

\footnotetext{
* Corresponding author: ogar@brstu.ru
} 
It was shown in [16] that if the branch of elastoplastic loading of the sphere of the kinetic indentation diagram has the form

$$
P=C h_{0}{ }^{\alpha}
$$

where $h_{0}$ is the maximum displacement in the contact, $C$ and $\alpha$ are constants, then the pressure distribution at the contact site is described by the expression

$$
p(r)=p_{0}\left(1-\frac{r^{2}}{a^{2}}\right)^{\beta},
$$

where $\beta=\alpha-1, p_{0}=p_{m}(\beta+1), p_{m}=P /\left(\pi a^{2}\right)$ is the average pressure in contact.

In view of considerable practical interest, the goal of this research is to determine the stressstrain state of a half-space under the action of a load described by expression (2) at $0<\beta<0.5$.

\section{Determination of displacements and stresses from the action of the applied load}

Using finite element modeling when introducting a sphere into an elastoplastic hardened half-space, the authors of [20] obtained that

$$
P=C\left(\varepsilon_{y}, n\right) h^{A\left(\varepsilon_{y}, n\right)},
$$

where $\varepsilon_{y}$ is the yield strain, $n$ is the hardening exponent. For $\varepsilon_{y}=0.001 \ldots 0.005$ and $n=0 . .0 .3: A\left(\varepsilon_{y}, n\right)=1.017 \ldots 1.277$. So in the equation (2) $\beta=0.017 \ldots 0.277$.

In the technical literature on the theory of elasticity and mechanics of contact introduction [21, 22, etc.], particular cases of the stress-strain state are considered for $\beta=0$ (uniform distribution) and for $\beta=0.5$ (hertz distribution). For $0<\beta<0.5$, data are not available due to the difficulties of obtaining analytical expressions when integrating products of power functions with fractional powers.

In a cylindrical coordinate system for an axisymmetric load, the Hooke relations have the form $[6,7]$ :

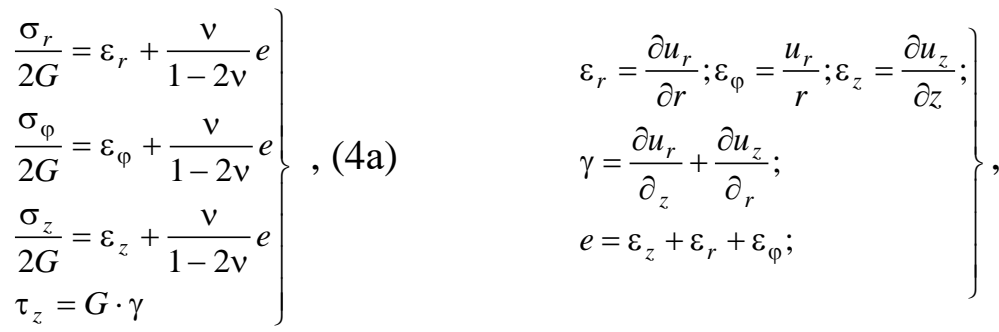

where $G=E / 2(1+v)$ is the shear modulus, $E$ is the elastic modulus, $v$ is the Poisson's ratio; $\sigma_{r}, \sigma_{\varphi}, \sigma_{z}$ - radial, circumferential and axial stresses; $u_{z}, u_{r}$ - axial and radial displacements. 
The stress and displacement from the action of normal pressure distributed over the half-surface area $S$ of the surface can be found by superposition using the results for the concentrated force. So, for radial and axial movements [7] we have

$$
\bar{u}_{r}=-\frac{1-2 v}{4 \pi G} \cdot \frac{P}{r}, \quad \quad \bar{u}_{z}=\frac{1-v}{2 \pi G} \cdot \frac{P}{r} .
$$

To calculate the displacements at the internal and external points from the load applied in a circular region, we use the approach proposed in [7, Fig. 3.5] for $\beta=0$ and $\beta=0.5$. Interpreting the pressure $p(t)$ at the internal point as a concentrated force, using expression (5), we find the normal displacement

$$
\bar{u}_{z}=\frac{\theta}{\pi} \iint_{s} p(s, \varphi) d s d \varphi,
$$

where $\theta=\left(1-v^{2}\right) / E$

Moving points of a loaded area is

$$
\bar{u}_{z}(r)=\frac{2 \theta p_{0}}{\pi a^{2 \beta}} \int_{0}^{\frac{\pi}{2}} \int_{s_{z}}^{s_{1}}\left(s_{1}-s\right)^{\beta}\left(s-s_{2}\right)^{\beta} d s d \varphi,
$$

where $s_{1}, s_{2}$ are the real roots of the equation $c^{2}-2 b s-s^{2}=\left(s_{1}-s\right) \cdot\left(s-s_{2}\right)$ for $c \geq 0$ :

$$
s_{1,2}=-b \pm \sqrt{b^{2}+c^{2}}, \quad c^{2}=a^{2}-r^{2}, \quad b=r \cos \varphi .
$$

As a result of integration, we obtain

$$
\bar{u}(r)=\theta p_{0} a \cdot 2^{2 \beta+1} \cdot \mathrm{B}(\beta+1, \beta+1){ }_{2} F_{1}\left(-\beta-\frac{1}{2}, \frac{1}{2} ; 1 ; \frac{r^{2}}{a^{2}}\right)
$$

where $\mathrm{B}\left(\alpha_{1}, \alpha_{2}\right)$ is the beta function, ${ }_{2} F_{1}(a, b, c, x)$ is the Gauss hypergeometric function

To determine the tangential displacement at the internal point, which due to axial symmetry must be radial, we use expression (5). The pressure at the elementary site at the point causes a tangential displacement at the internal point, equal to

$$
\frac{1-2 v}{1-v} \cdot \frac{\theta}{2 \pi} p(t) d s d \varphi
$$

The radial component of this movement is

$$
-\frac{1-2 v}{1-v} \cdot \frac{\theta}{2 \pi} p(t) \cos \varphi d s d \varphi
$$

The total tangential displacement at the internal point under the action of the total pressure distribution is

$$
\bar{u}_{r}(r)=\frac{1-2 \mu}{1-\mu} \cdot \frac{\theta p_{0}}{2 \pi a^{2 \beta}} \int_{0}^{2 \pi} \int_{0}^{s_{1}}\left(s_{1}-s\right)^{\beta}\left(s-s_{2}\right)^{\beta} d s \cos \varphi d \varphi .
$$


As a result of integration, we obtain

$$
\bar{u}_{r}(r)=\theta p_{0} a \cdot \frac{1-2 v}{1-v} \cdot\left[-2^{2 \beta+1} \mathrm{~B}(\beta+1, \beta+1) \cdot{ }_{2} F_{1}\left(-\beta-\frac{1}{2} ; \frac{1}{2} ; \frac{3}{2} ; \frac{r^{2}}{a^{2}}\right)+2\left(1-\frac{r^{2}}{a^{2}}\right)^{\beta} F_{\varphi}\left(\frac{r}{a}\right)\right],
$$

where $F_{\varphi}\left(\frac{r}{a}\right)=\int_{0}^{\frac{\pi}{2}}(-b(\varphi)+c(\varphi))_{2} F_{1}\left(-\beta, 1 ; \beta+2 ; \frac{-b(\varphi)+c(\varphi)}{-b(\varphi)-c(\varphi)}\right) \cos \varphi d \varphi$,

$$
b(\varphi)=\frac{r}{a} \cos \varphi, c(\varphi)=\left(1-\frac{r^{2}}{a^{2}} \sin ^{2} \varphi\right)^{\frac{1}{2}} .
$$

For a point outside the circular loading region, the integration limits over $\varphi$ are equal to $\varphi_{1}= \pm \arcsin \frac{a}{r}$. In this case, we have

$$
\begin{gathered}
\bar{u}_{z}(r)=\frac{2 \theta p_{0}}{\pi a^{2 \beta}} \int_{0}^{\varphi_{1}} \int_{s_{1}}^{s}\left(s_{2}-s\right)^{\beta}\left(s-s_{1}\right)^{\beta} d s d \varphi \\
\bar{u}_{z}(r)=\theta p_{0} a \frac{2^{2 \beta+1} \mathrm{~B}(\beta+1, \beta+1)}{\pi \cdot \frac{r}{a}} \cdot \mathrm{B}\left(\frac{1}{2}, \beta+\frac{3}{2}\right) \cdot{ }_{2} F_{1}\left(\frac{1}{2} ; \frac{1}{2} ; \beta+2 ; \frac{a^{2}}{r^{2}}\right)
\end{gathered}
$$

The components of deformations on the surface $\bar{\varepsilon}_{r}=\partial \bar{u}_{r} / \partial r$ and $\bar{\varepsilon}_{\varphi}=u_{r} / r$ can be determined from expressions (6) - (8). For points of the loaded region, we have

$$
\left.\begin{array}{l}
\frac{\bar{\sigma}_{r}}{p_{m}}=\frac{v}{1-v} \frac{\bar{\sigma}_{z}}{p_{m}}+\frac{1}{\theta p_{m} a}\left(\frac{\partial \bar{u}_{r}(\rho)}{\partial \rho}+\mu \frac{\bar{u}_{r}(\rho)}{\rho}\right) \\
\frac{\bar{\sigma}_{\varphi}}{p_{m}}=\frac{v}{1-v} \frac{\bar{\sigma}_{z}}{p_{m}}+\frac{1}{\theta p_{m} a}\left(\frac{\bar{u}_{r}(\rho)}{\rho}+\mu \frac{\partial \bar{u}_{r}(\rho)}{\partial \rho}\right) \\
\frac{\bar{\sigma}_{z}}{p_{m}}=-(\beta+1)\left(1-\rho^{2}\right)^{\beta}, \quad \rho=\frac{r}{a}
\end{array}\right\} .
$$

To determine the stresses inside the half-space, we use the expressions for the stresses from the concentrated force [7]

$$
\left.\begin{array}{l}
\sigma_{r}=\frac{P}{2 \pi}\left[(1-2 v) \cdot\left(\frac{1}{r^{2}}-\frac{z}{\rho r^{2}}\right)-\frac{3 z r^{2}}{\rho^{5}}\right] \\
\sigma_{\theta}=-\frac{P}{2 \pi}(1-2 v) \cdot\left(\frac{1}{r^{2}}-\frac{z}{\rho r^{2}}-\frac{z}{\rho^{3}}\right) \\
\sigma_{z}=-\frac{3 P}{2 \pi} \cdot \frac{z^{3}}{\rho^{5}}
\end{array}\right\} .
$$

Consider a ring element with an area of $2 \pi r d r$ and radius $r$. The load on the ring is $2 \pi r p(r) d r$. Integrating over the area of the circle for $\sigma_{z}$, we have 


$$
\begin{gathered}
\sigma_{z}=-\frac{3 z^{3} p_{0}}{a^{2 \beta}} \int_{0}^{a} r\left(a^{2}-r^{2}\right) \beta\left(r^{2}+z^{2}\right)^{-\frac{5}{2}} d r \\
\frac{\sigma_{z}}{p_{m}}=-(\beta+1) \cdot\left[{ }_{2} F_{1}\left(-\beta, 1 ;-\frac{1}{2} ;-\frac{z^{2}}{a^{2}}\right)+2 \sqrt{\pi} \frac{\Gamma(\beta+1)}{\Gamma(\beta+1,5)} \cdot\left(0,25-\beta^{2}\right) \frac{z^{3}}{a^{3}}\left(1+\frac{z^{2}}{a^{2}}\right)^{\beta+1}\right], \frac{z}{a} \leq 1,(11) \\
\frac{\sigma_{z}}{p_{m}}=-\frac{3}{2} \cdot \frac{a^{2}}{z^{2}} \cdot{ }_{2} F_{1}\left(1, \frac{5}{2} ; \beta+2 ;-\frac{a^{2}}{z^{2}}\right), \frac{z}{a} \geq 1 .
\end{gathered}
$$

From the expressions (10) it follows that

$$
\sigma_{\Sigma}=\sigma_{r}+\sigma_{\phi}+\sigma_{z}=-\frac{P}{\pi} \frac{(1+v) z}{\rho^{3}} .
$$

Substituting the pressure on the annular element $2 \pi r d r$, we obtain

$$
\begin{gathered}
\sigma_{\Sigma}=-\frac{2(1+v) z p_{0}}{a^{2} \beta} \int_{0}^{a} r\left(a^{2}-r^{2}\right) \beta\left(r^{2}+z^{2}\right)^{-\frac{3}{2}} d r \\
\frac{\sigma_{\Sigma}}{p_{m}}=-2(1+v)(1+\beta)\left[{ }_{2} F_{1}\left(-\beta, 1 ; \frac{1}{2} ;-\frac{z^{2}}{a^{2}}\right)-\frac{\sqrt{\pi} \Gamma(\beta+1)}{\Gamma(\beta+0,5)} \cdot \frac{z}{a}\left(1+\frac{z^{2}}{a^{2}}\right)^{\beta-0.5}\right], \frac{z}{a} \leq 1,(12) \\
\frac{\sigma_{\Sigma}}{p_{m}}=-(1+v) \frac{a^{2}}{z^{2}} \cdot{ }_{2} F_{1}\left(1, \frac{3}{2} ; \beta+2 ;-\frac{a^{2}}{z^{2}}\right), \quad \frac{z}{a} \geq 1 .
\end{gathered}
$$

On the $\mathrm{z}$ axis, we have $\sigma_{r}=\sigma_{\varphi}$; therefore, from (11) and (12) we have

$$
\sigma_{r}=\sigma_{\varphi}=0,5\left(\sigma_{\Sigma}-\sigma_{z}\right)
$$

In the loading region and on the $\mathrm{z}$ axis, the stresses $\sigma_{r}, \sigma_{\varphi}, \sigma_{z}$ are main, with $\sigma_{1}=\sigma_{z}$ $, \sigma_{2}=\sigma_{\varphi}, \sigma_{3}=\sigma_{r}$.

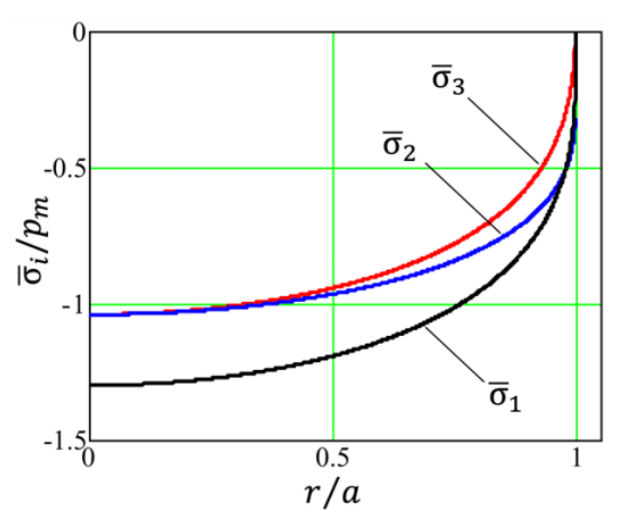

Fig. 1. The main stresses on the contact area at $\beta=0.3$

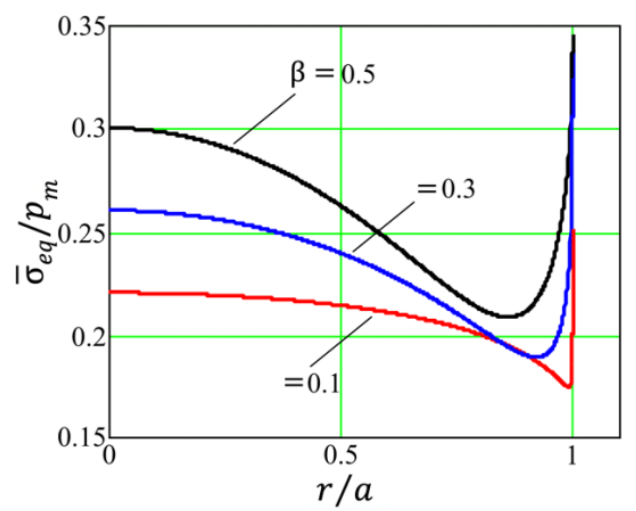

Fig. 2. Equivalent stresses at the contact area 
In fig. 1 shows the principal stresses on the loaded surface at $\beta=0.3$. In fig. 2 shows equivalent stresses on a loaded surface. In fig. 3 shows the principal $(a)$ and equivalent $(b)$ stresses on the $z$ axis for the same values of $\beta$. Equivalent stresses in the center of the circular region are proportional to $(1+\beta), \sigma_{\mathrm{eq}}(0) / p_{m}=0,2(1+\beta)$.

\section{Plasticity criterion}

Of interest is the load at which the plastic flow begins under conditions of a complex stress state, which is determined by the yield strength $\sigma_{Y}$ of the softer tensile material, which is part of the corresponding yield stress (plasticity). The closest coincidence with the experimental data on the indentation of indenters into elastoplastic media was shown by the Mises energy theory of shear strain and the Tresca theory of maximum tangential stresses.

According to Mises:

$$
\sigma_{Y}=\frac{1}{\sqrt{2}} \sqrt{\left(\sigma_{1}-\sigma_{2}\right)^{2}+\left(\sigma_{2}-\sigma_{3}\right)^{2}+\left(\sigma_{3}-\sigma_{1}\right)^{2}} .
$$

According to Tresca:

$$
\sigma_{Y}={ }_{\max }\left(\left|\sigma_{1}-\sigma_{2}\right|,\left|\sigma_{2}-\sigma_{3}\right|,\left|\sigma_{3}-\sigma_{1}\right|\right)
$$

The difference between the two criteria is small, so it is advisable to use the Tresca criterion because of its algebraic simplicity.

a)

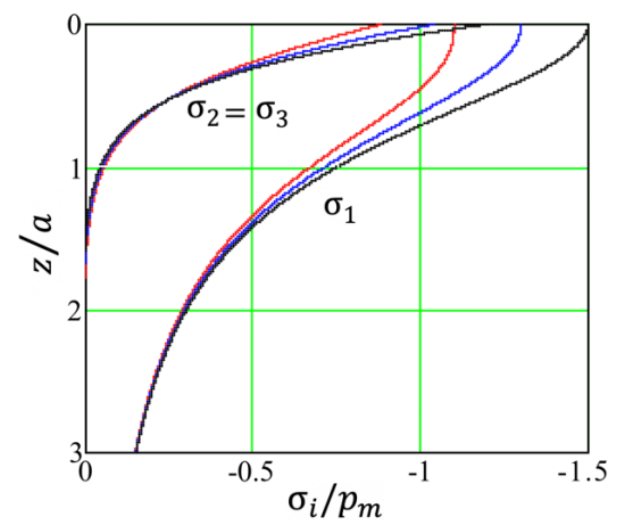

b)

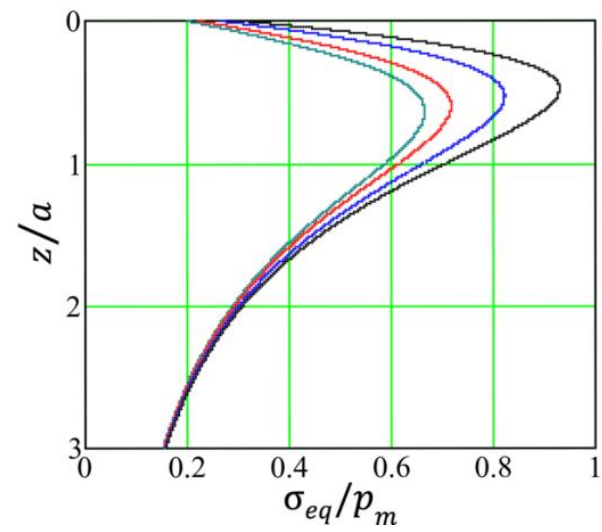

Fig. 3. The main stresses (a) and equivalent (b) stresses on the $z$ axis at $\beta$ values: (curves from left to right) $0.1 ; 0.3 ; 0.5$

As follows from fig. 3, the maximum values of equivalent stresses occur on the $Z$ axis. According to the criterion of the maximum tangential stress of Tresca, plastic deformation on the $Z$ axis corresponds to the equivalent stress

$$
\sigma_{\mathrm{eq}}=\left|\sigma_{3}-\sigma_{1}\right|=2 \tau_{1 \max }=\sigma_{Y}
$$

For $\beta=0,5$, the maximum contact pressure at which $\sigma_{\mathrm{eq}}=\sigma_{Y}$, we have 


$$
p_{0}=1.613 \sigma_{Y}
$$

The maximum contact pressure at which plastic deformation begins can be represented by the expression

$$
p_{0 p}=K_{Y} \sigma_{Y}
$$

where $K_{Y}$ is a constant.

In contact problems of tribology, a $K_{Y}$ value close to 3 is used. Obviously, the onset of plastic deformation on the contact surface is of interest in this case, which can explain that the value $K_{Y}>1.613$.

The equivalent stress $\sigma_{\text {eq }}(\rho)$ has the greatest value at the edge of the loading region, where it slightly exceeds the value of $\sigma_{\text {eq }}(0)$ in the center of the loading region. It is convenient to imagine the equivalent stress at the edge of the loading region:

$$
\sigma_{\text {eq }}(1)=K_{\sigma} \cdot \sigma_{\text {eq }}(0) \text {. }
$$

For $\beta=0,5$, according to the energy theory of shear strain, $K_{\sigma}=1.16$, according to the theory of maximum shear stresses $K_{\sigma}=1.33$, and according to the theory of maximum reduced stress $K_{\sigma}=1$. For other values of $\beta, K_{\sigma}$ takes different values (fig. 4).

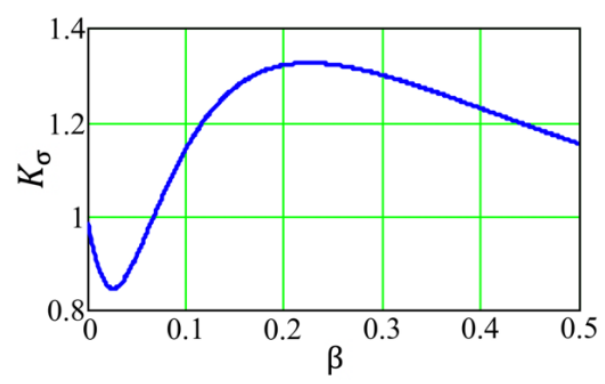

Fig. 4. The dependence of the parameter $K_{\sigma}$ on the exponent $\beta$

An analysis of expressions (11), (12), and (13) at $\rho=0$ implies

$$
\sigma_{e q}=\frac{1-2 v}{2} K_{\sigma} p_{0}=\sigma_{Y}
$$

Then the maximum contact pressure in the loading region at which plastic deformation takes place, is

$$
p_{0 p}=\frac{2 \sigma_{Y}}{(1-2 v) K_{\sigma}}
$$

then $\bar{K}_{Y}=\frac{2}{(1-2 v) K_{\sigma}}$.

This expression is valid for all values of $\beta$. 


\section{Conclusion}

1. Expressions are obtained that are of practical interest in determining the stress-strain state when loading an elastoplastic half-space with an axisymmetric load of the form $p(r)=p_{0}\left(1-r^{2} / a^{2}\right)^{\beta}$, where $0 \leq \beta \leq 0.5$. For $\beta=0$ and $\beta=0.5$, there is complete agreement with the data [21, 22].

2. The conditions of the onset of plastic deformation in the near-surface layer and on the surface of the half-space are considered. The maximum equivalent stresses are achieved in the surface layer on the axis of symmetry. The values of these stresses are determined by expressions (12) - (15). The maximum values for $v=0.3$ vary from $0.93 p_{m}$ at depth $0.48 a$ to $0.666 p_{m}$ at depth as $\beta$ change from 0.5 to 0 (Fig. 3). The condition for the appearance of plastic deformations on the surface is described by expression (16).

3. As follows from fig. 4 , with $0<\beta<0.068$ equivalent stresses at the edge of the loading platform less than equivalent stresses in the center of the platform.

\section{References}

1. J.A. Greenwood, J.B.R. Williamson, Proc. Roy.Soc. A295, 301 (1966)

2. 2. N.B. Demkin, Contact of rough surfaces (1970)

3. P. Ogar, S. Belokobylsky, D. Gorokhov, Contact and Fracture Mechanics (2018)

4. A.A. Lankov, Friction and lubrication in machines and mechanisms, 3, 3 (2009)

5. H. Gheadnia, S. A. Pope, R. L. Jackson, D. B. Marghitu, Tribiology International, 93, Part A, 78 (2016)

6. H. Ghaednia, X. Wang, S. Saha, Y. Xu, A. Sharma, R. L. Jackson, ASME. Appl. Mech. Rev., 69(6): 060804 (2017)

7. N.A. Voronin, J. Friction \& Wear, 24 (1) 16 (2003)

8. M.M. Matlin, S.L. Lebskij, A.I. Mozgunova, Regularities of Elastoplastic Contact in Problems of Surface Plastic Hardening (2007)

9. R. Hill, B. Storakers, A. B. Zdunek, Proc. Royal Soc., A 423, 301 (1989)

10. J.H. Ahn, D. Kwon, Mater. J. Res., 16 (11), 3170 (2001)

11. B. Taljat, G. M. Pharr, Int. J. Solids and Struct., 41, 3891 (2004)

12. .H. Kim, B.W. Lee., Y. Choi, D. Kwon, Mat. Sci. Eng., A 415, 59 (2006)

13. R.L.,Jackson, L. Kogut, ASME J.Tribol., 128 (1), 209 (2006)

14. M.M. Matlin, E.N. Kazankina, V.A. Kazankin, J. Friction \& Wear, 32 (2) 140 (2011)

15. E. Olsson, P.L. Larsson, Int. J. Solids Struct., 81, 23 (2016)

16. P.M. Ogar, V.A. Tarasov, Adv. Mat. Res., 664, 625 (2013)

17. P.M. Ogar, D.B. Gorokhov, A.S. Kozhevnikov, Proc. 2nd Int. Conf. on Modelling, Identification and Control (MIC 2015)

18. P. Ogar, D. Gorokhov, L. Mamaev, V. Fedorov, Adv. Eng. Res, 158, 313 (2018)

19. P. Ogar, D. Gorokhov, E. Ugryumova, MATEC Web of Conf., 129, 06016 (2017)

20. J.-M. Collin, G. Mauvoisin, P. Pilvin, Materials and Desing. 31, 636 (2010)

21. S.P. Timoshenko, J. Goodyer, Theory of Elasticity (1979)

22. K.L. Johnson, Contact mechanics (1981) 